\title{
Genotipificación a gran escala en cáncer de pulmón
}

\author{
Large-scale genotyping of lung cancer
}

\author{
Noemí Reguart ${ }^{1}$, Andrés Felipe Cardona ${ }^{2,3}$, Mauricio Cuello ${ }^{4}$ Óscar Arrieta ${ }^{5}$
}

Departamento de Oncología Médica, Sección Oncología Torácica, Hospital Clínico y Provincial de Barcelona (Barcelona, España).

2'Grupo Oncología Clínica y Traslacional, Instituto de Oncología, Fundación Santa Fe de Bogotá (Bogotá, Colombia).

${ }^{3}$ Fundación para la Investigación Clínica y Molecular Aplicada del Cáncer (FICMAC); investigador asociado ONCOLGroup.

${ }_{5}^{5}$ Clínica de Oncología Torácica y Laboratorio de Oncología Experimental, Instituto Nacional de Cancerología de México (INCAN) (México D.F., México).

En los últimos 30 años, hemos observado, silentes, el incremento poético y progresivo de la tipificación a gran escala del genoma humano y de su vertiente tumoral'. Hoy sabemos que el cáncer incluye más de 100 enfermedades genéticas diferentes, con diversos factores de riesgo y epidemiología, que se caracterizan por la proliferación incontrolada de células capaces de invadir tejidos más allá de los límites normales, generando metástasis en órganos distantes ${ }^{2}$. Las fuentes de variabilidad genética asociadas a la enfermedad se deben en parte a la presencia de mutaciones que constituyen cambios estables en la secuencia de bases de un gen, que no se deben a segregación o recombinación durante la meiosis.

Estas alteraciones pueden ser sustituciones, deleciones, inserciones, inversiones y translocaciones, al igual que silenciosas o neutras, deletéreas o letales, sin sentido o con sentido equívoco. Algunas se consideran conductoras cuando confieren ventajas a la célula tumoral para evolucionar en el proceso de crecimiento, permitiéndole la selección positiva de la neoplasia. Por el contrario, muchas son pasajeras, porque no modifican el curso biológico de la enfermedad. Aproximadamente, 100.000 mutaciones somáticas se han reportado en el último cuarto de siglo desde que la primera se encontrara en el HRAS.

El cáncer de pulmón constituye una de las enfermedades más complejas, ya que contiene más de 33.000 alteraciones, de las que cerca de 500 se consideran dominantes ${ }^{3,4}$. Globalmente, se están desarrollando cuatro esfuerzos para valorar más de 25.000 muestras que permitirán discriminar las mutaciones más relevantes en los adenocarcinomas, carcinomas escamosos y de célula pequeña (Lung Cancer Mutation Consortium, Institut National du Cancer, Collaborative Advanced Stage Tissue Lung Cancer, Cancer Research UK).
Recientemente, Sequist y colaboradores clasificaron 531 carcinomas de pulmón (431 utilizando la tecnología Snapshot versión 1 y empleando la versión 2); casi todas las muestras (528) fueron valoradas por FISH para el gen de fusión ALK/EML4. La mediana de edad de la población fue de 64 años, e incluyó un 58\% de mujeres y $92 \%$ de caucásicos. El $24 \%$ eran nunca fumadores, la histología dominante fue el adenocarcinoma (81\%) y el tiempo para la evaluación biológica fue de 2,8 semanas ${ }^{5}$. Se encontró alguna mutación en el $51 \%$ de las muestras, mientras el complemento se consideró negativo (figura 1); 25 pacientes tuvieron mutaciones complejas (2 o 3) y la distribución para los cambios más representativos fue del 13\%, 24\%, 5\%, 5\%, 4\%, 2\%, $2 \%$ y $1 \%$ para el EGFR, KRAS, ALK, TP53, PI3KCA, $\beta$-catenina, BRAF y NRAS, respectivamente. También se encontraron dos pacientes con alteraciones en el HER2 y una mutación del IDH1 (R132H).

El examen de la distribución demográfica y otras correlaciones clínicas con el genotipo demostró que los pacientes con mutaciones del EGFR solían ser mujeres de origen asiático que presentaban adenocarcinomas.

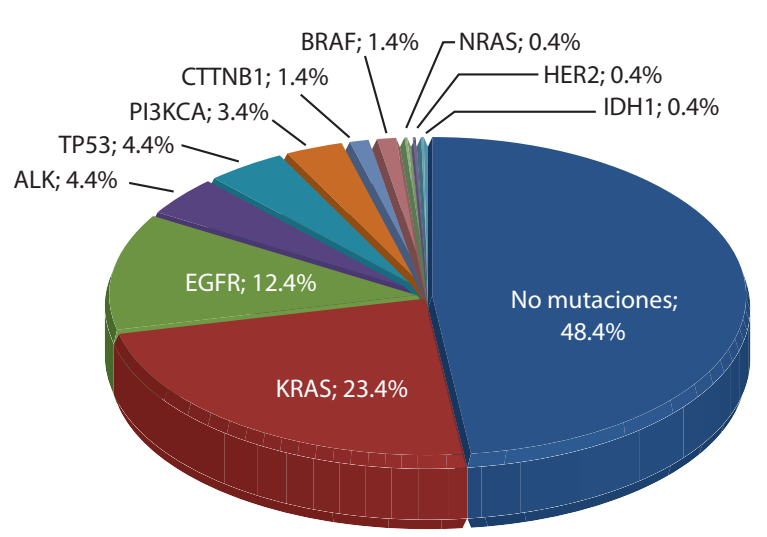

Figura 1. Distribución de las alteraciones genómicas dominantes en el cáncer de pulmón. 
En esta cohorte, las mutaciones del KRAS estuvieron asociadas con la etnia caucásica ( $p=0.02$ ), con el fenotipo glandular ( $p=0.004)$ y con una presentación más temprana de la enfermedad ( $p=0.002$ ). Las translocaciones del ALK se correlacionaron con una menor edad $(p<0.001)$ y con una mayor extensión de la patología $(p$ $=0.06$ ), mientras los cambios en el PI3KCA ocurrieron exclusivamente en los carcinomas escamosos.

Al igual que en otras series, la historia de exposición al humo por combustión de tabaco fue uno de los discriminantes primarios, puesto que, cuando esta fue menor, se documentó una asociación positiva con los cambios genómicos en el EGFR, ALK y $\beta$-catenina. En contraposición, los fumadores presentaron una mayor proporción de mutaciones en KRAS y NRAS. La supervivencia global (SG) para toda la población fue de 21.7 meses, con una notable reducción temporal en la población de mutados para el KRAS $(p=0.04)$.

Por el contrario, los sujetos portadores de mutaciones en el EGFR tuvieron una SG de 34.3 meses versus 20 meses para la población silvestre. El punto más sobresaliente del estudio fue que el $38 \%$ de los pacientes con un genotipo potencialmente modulable (EGFR, KRAS, ALK, BRAF, PI3KCA, HER2) fueron incluidos en algún estudio de terapia blanco ${ }^{5}$. Un análisis reciente realizado en China, en el que se utilizó un panel de genotipificación similar en pacientes nunca fumadores con adenocarcinoma, documentó una proporción impresionante (90\%) de mutaciones en el EGFR, KRAS, ALK O HER2.

Estos hallazgos sustentan la importancia de valorar las diferencias étnicas existentes en el cáncer de pulmón de célula no pequeña (CPCNP) ${ }^{6}$. En el pasado, Arrieta y colaboradores encontraron datos diferentes tras integrar los genotipos de más de 1.000 sujetos evaluados en cuatro países de América Latina ${ }^{7}$. Durante el último encuentro anual de la Sociedad Americana de Oncología Clínica (ASCO, por su sigla en inglés), se abrieron puertas similares para los carcinomas escamosos 8,9 .

En el Memorial Sloan-Kettering Cancer Center, se evaluaron 40 especímenes de carcinomas escamosos de pulmón, utilizando la plataforma SQ-MAP (Multiplex PCR) más la evaluación rutinaria de la amplificación del FGFR1 valorada por FISH (definido como CEP8 mayor o igual a 2 en más del $10 \%$ de las células), inmunohistoquímica para la pérdida de PTEN y Sequenom
MassARRAY para el PI3KCA ${ }^{8}$. También se incorporó la secuenciación exómica (Agilent Sure Select/lon Torrent) de un panel de más de 80 oncogenes y genes supresores de tumor previamente reconocidos en cáncer de pulmón. La información estuvo disponible en más de 28 sujetos en los que se encontró una tasa de mutaciones del 60\% (IC95\% 37\%-75\%), siendo las más importantes la amplificación del FGFR1 (25\%), la pérdida completa del PTEN (20\%), las mutaciones del PI3KCA (11\%) y las del KRAS (4\%).

Por otra parte, el TCGA reclutó 178 pacientes con carcinomas escamosos en los que se realizó una amplia secuenciación exómica que documentó 13 genes alterados con extrema frecuencia (siendo los más importantes TP53, CDKN2A, PTEN, KEAP1, NFE2L2, RB1, NOTCH1, NF1, PI3K y AKT). En casi todas las muestras, se evidenció una pérdida universal del TP53 y del CDKN2A, mientras los cambios en NFE2L2/KEAP1 y PI3K/ AKT estuvieron en el $35 \%$ y $43 \%$ de los tumores, respectivamente. La valoración de la expresión del mARN distinguió cuatro subtipos tumorales, cada uno con un perfil mutacional típico; el primero, denominado clásico $(37 \%)$, presentó alteraciones en NFE2L2, KEAP1 y FGFR1, y una elevada metilación debido a la exposición al tabaco. El segundo, llamado subtipo basal (24\%), estuvo caracterizado por las alteraciones en las quinasas relacionadas con el FGFR1. El tercero, conocido como secretor (24\%), tuvo cambios en el PDGFRA; y el último, denominado primitivo (15\%), se asoció con mutaciones en RB1. De forma global, los carcinomas escamosos tuvieron una tasa de modificaciones genómicas del 72\%, tres cuartas partes de ellas potencialmente modificables con medicamentos en curso de investigación ${ }^{9}$.

Hace poco, Pérez-Moreno y colaboradores recordaron las características típicas del fenotipo de los carcinomas escamosos, incluyendo la expresión de p63 y de la porción truncada N-terminal P40/TTF110. En concordancia, la Organización Mundial de la Salud (OMS) reconoce cuatro variantes para este subtipo del CPCNP, considerando un patrón de células claras, otro de células pequeñas, otro papilar y el basaloide. La variante papilar demuestra un crecimiento endobronquial obstructivo que en algunos casos está limitado a la diseminación intraepitelial donde la invasión suele ser difícil de valorar.

Por otro lado, el histotipo basaloide se presenta con proliferación de células pluripotenciales de origen 
Tabla 1. Paralelo de las alteraciones genómicas entre el carcinoma escamoso y el adenocarcinoma de pulmón

\begin{tabular}{|c|c|c|c|c|}
\hline Anormalidad genética & Gen & Adenocarcinoma & Carcinoma escamoso & Terapia \\
\hline Mutación TP53 & $17 \mathrm{p} 13.1$ & $36 \%$ & $51 \%$ & Onix 015, SCH500 \\
\hline Amplificación PI3KCA & $3 q 26.3$ & $6 \%$ & $33 \%$ & - \\
\hline Amplificación SOX2 & $3 q 26.3-q 27$ & Muy rara & $23 \%$ & ZF-4203SKD \\
\hline Amplificación FGFR1 & $8 p 12$ & $1 \%$ & $22 \%$ & BGJ398, AZD4547, E-3810, FP-1039, TKI258 \\
\hline Mutación PTEN & $10 q 23.3$ & $2 \%$ & $10 \%$ & - \\
\hline Amplificación MET & $7 q 31.1$ & $3-21 \%$ & $3-21 \%$ & - \\
\hline Pérdida PTEN & $10 \mathrm{q} 23.3$ & $8-20 \%$ & $8-20 \%$ & - \\
\hline Mutación KRAS & $12 \mathrm{p} 12.1$ & $21 \%$ & $6 \%$ & Zelumetinib \\
\hline EGFRvill (mutación) & $7 p 12$ & Muy rara & $5 \%$ & Rindopepimut \\
\hline Mutación LKB1 & $19 p 13.3$ & $23 \%$ & $5 \%$ & - \\
\hline Mutación DDR2 & $1 \mathrm{q} 23.3$ & $1 \%$ & $4 \%$ & Dasatinib \\
\hline Sobreexpresión HER2 & $17 q 11.2-q 12,17 q 21$ & $5-9 \%$ & $3-5 \%$ & Trastuzumab \\
\hline Mutación PI3KCA & $3 q 26.3$ & $3 \%$ & $3 \%$ & PF-04691502, XL147, BKM120, BYL719, XL765, PX866, GDC-0941 \\
\hline Mutación BRAF & $7 \mathrm{p} 34$ & $1-3 \%$ & $2 \%$ & GSK2118436, vemurafenib \\
\hline Mutación EGFR & $7 p 12$ & $10-15 \%$ & $<5 \%$ & Erlotinib, gefitinib, afatinib, dacomitinib, XL186 \\
\hline Mutación AKT1 & $14 q 32.32$ & Muy rara & $1 \%$ & MK2206, GDC-0068 \\
\hline Mutación MET & $7 q 31.1$ & $2 \%$ & $1 \%$ & Crizotinib, XL184, MetMAb, Arq197 \\
\hline Mutación HER2 & $17 q 11.2-q 12,17 q 21$ & $2 \%$ & $1 \%$ & MGAH22, afatinib, lapatinib \\
\hline EML4-ALK & $2 \mathrm{p} 21,2 \mathrm{p} 23$ & $2-7 \%$ & $1 \%$ & Crizotinib \\
\hline
\end{tabular}

bronquial con mínima diferenciación escamosa y positividad para las citoqueratinas 5/6, CK1, CK10 y CK14, reconocida por el anticuerpo CK34ßE12. A pesar de que la terapia estándar para los carcinomas escamosos sigue siendo la quimioterapia y de que hasta el momento no hay ningún estudio fase III que esté valorando terapias dirigidas para esta subpoblación específica, hay una pléyade de intervenciones en evaluación dirigidas en especial contra el FGFR1, DDR2, MET y PI3K/AKT. Por el momento, tendremos que aguardar por la evolución de estos estudios. La tabla 1 incluye un paralelo de las frecuencias relativas para las mutaciones dominantes de los carcinomas escamosos versus los adenocarcinomas y sus tratamientos potenciales.

Los estudios mencionados a lo largo del presente editorial han abierto la caja de Pandora mostrándonos una nueva historia para la biología y la evolución clonal del CPCNP. Al igual que en la teogonía de Hesíodo, tendremos que urdir un engaño prometeico en contra del "mal" con la intención de modificar el daño creciente que las neoplasias ejercen sobre nuestra envejecida humanidad.

\section{Referencias}

1. Stratton MR, Campbell PJ, Futreal PA. The cancer genome. Nature. 2009;458(7239):719-24.

2. Krontiris TG, Cooper GM. Transforming activity of human tumor DNAs. Proc Natl Acad Sci USA. 1981;78(2):1181-4.

3. Dias-Santagata D, Akhavanfard S, David SS, Vernovsky K, Kuhlmann G, Boisvert SL, et al. Rapid targeted mutational analysis of human tumours: a clinical platform to guide personalized cancer medicine. EMBO Mol Med. 2010;2(5):146-58.

4. Pao $W$, Kris MG, lafrate AJ, Ladanyi M, Jänne PA, Wistuba II, et al. Integration of molecular profiling into the lung cancer clinic. Clin Cancer Res. 2009;15(17):5317-22.

5. Sequist $L V$, Heist RS, Shaw AT, Fidias $P$, Rosovsky R, Temel JS, et al. Implementing multiplexed genotyping of non-smallcell lung cancers into routine clinical practice. Ann Oncol. 2011;22(12):2616-24.

6. Sun $Y$, Ren $Y$, Fang Z, Li C, Fang R, Gao B, et al. Lung adenocarcinoma from East Asian never-smokers is a disease largely defined by targetable oncogenic mutant kinases. J Clin Oncol. 2010;28(30):4616-20.

7. Arrieta $O$, Cardona AF, Federico Bramuglia G, Gallo A, Campos-Parra AD, Serrano S, et al.; CLICaP. Genotyping non-small cell lung cancer (NSCLC) in Latin America. J Thorac Oncol. 2011;6(11):1955-9.

8. Paik PK, Hasanovic A, Wang L, Rekhtman N, Ladanyi M, Kris MG. Multiplex testing for driver mutations in squamous cell carcinomas of the lung. J Clin Oncol. 2012;30 (suppl; abstr 7505).

9. Govindan R, Hammerman PS, Hayes DN, Wilkerson MD Baylin $\mathrm{S}$, Meyerson M, on behalf of the Cancer Genome Atlas (TCGA) Group. Comprehensive genomic characterization of squamous cell carcinoma of the lung. J Clin Oncol. 2012;30 (suppl; abstr 7006).

10. Pérez-Moreno P, Brambilla E, Thomas R, Soria JC. Squamous cell carcinoma of the lung: molecular subtypes and therapeutic opportunities. Clin Cancer Res. 2012;18(9):2443-51. 